phiinae zu den Cerberinae eine enge Beziehung haben.

Die einzelnen Triben, Subtriben und Gattungen zeigen demnach neben gleichen morphologischen Merkmalen eine entsprechende chemische Synthesefähigkeit, die sich im gleichzeitigen Vorkommen biogenetisch verwandter Inhaltsstoffe äußert.

Die Familie der Loganiaceae ist nun durch das Vorherrschen der Alkaloidbildung und das völlige Zurücktreten der Cardenolidglykoside gekennzeichnet. Lediglich die Buddleioideae enthalten keine Alkaloide, sind aber erst wenig bearbeitet. Auf Grund ihrer Inhaltsstoffe könnte man diese Unterfamilie aus den Loganiaceae ausgliedern. Es ist interessant, $\mathrm{da}$ bei der morphologischen Bearbeitung dieser Familie bereits ähnliche Vorschläge gemacht worden

96 R. H änsel u. L. H örh a m m e r, Arch. Pharmaz. Ber. dtsch. pharmaz. Ges. 287./59. 189 [1954]; L. H ö r h a m m e r u. R. H äm e l, Arch. Pharmaz. Ber. dtsch. pharmaz. Ges. 288, 153 [1955].

97 R. H e g n a u e r, Pharmac. Acta Helvetiae 29, 203 [1954]. sind $^{1}$. Durch das Loganin (identisch mit Meliatin) zeigen die Loganiaceae eine Verbindung zu den Menyanthaceae.

Trotz der Schwierigkeiten einer kritischen Beurteilung scheint uns bei Betrachtung einiger sogenannter sekundärer Pflanzeninhaltsstoffe in der Ordnung der Contortae eine Beziehung zwischen der chemischen Synthesefähigkeit höherer Pflanzen und deren Morphologie deutlich erkennbar zu sein. Eine Stütze unserer Anschauungen sehen wir darin, daß in neuerer Zeit eine Reihe anderer Autoren, wie $\mathrm{H}$ äns el ${ }^{96}$, $\mathrm{H}$ e g n a u e r ${ }^{97}, \mathrm{H}$ ö r h a m m er ${ }^{96}, \mathrm{M}$ a n s k e ${ }^{98}$ und W e evers ${ }^{99}$ ähnliche Gedankengänge durch experimentelle Untersuchungen zu beweisen versuchen.

Wir danken der Deutschen Forschungsgemeinschaft auch an dieser Stelle für die Gewährung einer Sachbeihilfe.

98 R. H. F. M a n ske, Imp. Bur. Pastures Forage Crops, Herbage Pub. Series, Bull. 27, 9 [1940], ref. C. A. 34, 2415, 5237 [1940].

99 T. W e e ver s, Blumea, Tijdschr. systematiek Geografie planten 5, 412 [1943].

\title{
Der Quantenbedarf bei der CO-Hämoglobinspaltung durch Licht
}

\section{Von Rudolf Burberg}

\author{
Aus dem Max-Planck-Institut für Virusforschung, Tübingen \\ (Z. Naturforschg. 10 b, 503-509 [1955]; eingegangen am 1. Juni 1955)
}

\begin{abstract}
Bei einer photochemischen Reaktion wird das Verhältnis von absorbierten Lichtquanten zum erfolgten photochemischen Umsatz als Brutto-Quantenbedarf, das Verhältnis von den an der Photoreaktion selbst beteiligten Lichtquanten zum erfolgten photochemischen Umsatz als Netto-Quantenbedarf definiert.

Es wird gezeigt, wie man aus Oszillographen-Schirmbild-Aufnahmen des Reaktionsbeginns Brutto- und Netto-Quantenbedarf einer photochemischen Reaktion bestimmen kann.

Die Methode wird dazu benutzt, die lichtempfindliche Abspaltung des CO-Moleküls vom CO-Hämoglobin zu untersuchen. Der Brutto-Quantenbedarf liegt je nach Salz- und Wasserstoffionenkonzentrationen zwischen $n=2$ und 5; die Werte stimmen mit Messungen von $\mathrm{B}$ ü ch e ${ }^{1}$ überein, die nach einer von W a r b u r g angegebenen Methode gemacht wurden. Für den Nettoquantenbedarf hat sich in allen Fällen $n^{*}=1$ ergeben.
\end{abstract}

$\mathrm{B}_{\mathrm{s}}$ ei Kohlenmonoxyd-Hämoglobin und anderen COHäm-Verbindungen ${ }^{2}$ können durch Bestrahlung mit Licht die CO-Moleküle abgespalten werden.

Wenn man nach dem Quantenbedarf fragt, den diese photochemischen Reaktionen benötigen, dann ist zunächst zu bemerken, daß man das Wort Quantenbedarf in verschiedenem Sinn gebrauchen kann. Um Mißverständnisse auszuschließen, möchte ich deshalb zwischen dem Brutto-Quantenbedarf und dem Netto-Quantenbedarf einer photochemischen Reak-

1 Th. B ü ch e r, Biochem. Z. 311, 163 [1942]. tion unterscheiden. Der Brutto-Quantenbedarf $n$ ist die Zahl der Lichtquanten, die vom CO-Hämoglobin je abgespaltenes CO-Molekül absorbiert worden sind.

Wenn nun zum Beispiel der Brutto-Quantenbedarf $n=4$ ist, dann kann der Reaktionsmechanismus so gestaltet sein, daß die Abspaltung erst in 4 Schritten über 3 photoempfindliche Zwischenprodukte erfolgt, oder daß ein Teil der Energie von den 4 Quanten irgendwelchen anderen Prozessen im Molekül zu-

2 O. W a r burg, Schwermetalle als Wirkungsgruppen von Fermenten, Saenger, Berlin 1946. 


\begin{tabular}{|c|c|c|c|c|c|}
\hline & $\begin{array}{r}\mathrm{Br} \\
\text { Quante } \\
p_{\mathrm{H}}=7,6\end{array}$ & $\begin{array}{l}\text { utto- } \\
\text { abed } \\
8,1 \\
\end{array}$ & $\begin{array}{r}\text { rf } n \\
9,0\end{array}$ & $\begin{array}{l}\text { Zahlder } \\
\text { Häme je } \\
\text { Molekül }\end{array}$ & $\begin{array}{l}\text { Mol.- } \\
\text { Gew. }\end{array}$ \\
\hline $\begin{array}{l}\text { CO-Hämoglobin } \\
\text { (Pferd) }\end{array}$ & 3,4 & 3,7 & 4,2 & 4 & 66000 \\
\hline $\begin{array}{l}\text { CO-Halbhämo- } \\
\text { globin (Pferd) }\end{array}$ & 2,2 & 2,7 & 2,8 & 2 & 33000 \\
\hline $\begin{array}{l}\text { CO-Myoglobin } \\
\text { (Pferd) }\end{array}$ & & 1,1 & & 1 & 17000 \\
\hline
\end{tabular}

Tab. 1. Die Meßergebnisse von B ü c h e r für den BruttoQuantenbedarf $n$ bei verschiedenen CO-Häm-Eiweißverbindungen und verschiedenem $p_{\mathrm{H}}$-Wert. Wellenlänge des spaltenden Lichts $\lambda=546 \mathrm{~m} \mu$.

geführt wird, die mit der photochemischen Abspaltung des CO nichts zu tun haben, und nur 1, 2 oder 3 Quanten die photochemische Abspaltung in 1, 2 bzw.3 Schritten bewirken. Wir müssen also zwischen unmittelbar am photochemischen Prozeß beteiligten Quanten (H a u s s e ${ }^{3}$ nennt sie ,aktive Quanten“) und am photochemischen Prozeß nicht beteiligten Quanten unterscheiden. Dann ist der Netto-Quantenbedarf $n^{*}$ die Zahl der an der photochemischen Reaktion unmittelbar beteiligten (aktiven) Quanten je abgespaltenes CO-Molekül.

Der Brutto-Quantenbedarf ist schon von B ü$\mathrm{che}^{1}$ nach einer von Warburg ${ }^{2}$ angegebenen Methode gemessen worden. Seine Ergebnisse sind in Tab. 1 zusammengefaßt.

$\mathrm{Da}$ beim CO-Hämoglobin, abweichend von allen anderen CO-Häm-Verbindungen (s. l.c. $\left.{ }^{2}\right)$ der BruttoQuantenbedarf größer als 1 ist, soll in vorliegender Arbeit die Frage entschieden werden, ob ein gröBerer Netto-Quantenbedarf vorliegt, d.h. die Reaktion eine eigentliche Mehrquantenreaktion ist, oder ob der Netto-Bedarf $n^{*}=1$ ist, also die CO-Hämoglobin-Spaltungsreaktion ein Einquantenprozeß ist wie man es wohl bei der CO-Myoglobin-Spaltungsreaktion und den anderen CO-Häm-Spaltungsreaktionen annehmen darf, für die alle nach Untersuchungen von $\mathrm{W}$ arburg und Mitarbb. $n=1$ gefunden worden ist ${ }^{2}$.

Den Netto-Quantenbedarf kann man dadurch feststellen, daß man den zeitlichen Verlauf des Reaktionseinsatzes untersucht: Wir haben deswegen die $\mathrm{Zu}-$ sammensetzung des Reaktionsgemisches vom Beginn

3 K. H. H a u s s e r, Z. Naturforschg. 5 a, 41 [1950].

4 Hämoglobin von Pferd und Rind in starker Salzlösung zerfällt in zwei gleiche Teile, sie sind in Tab. 1 mit Halbhämoglobin bezeichnet; vgl. hierzu J. S te i n h a r d t , J. biol. Chemistry 123, 543 [1938]. der Belichtung an fortlaufend spektrophotometrisch bestimmt und auf der Y-Achse des Schirmbildes eines mit waagrechter Zeitablenkung versehenen Oszillographen zur Anzeige gebracht.

Hat die Reaktion einen Netto-Bedarf von mehreren Quanten, müssen nach Einschalten der Lichtquelle erst die Zwischenprodukte gebildet werden, bevor das Endprodukt in Erscheinung treten kann: Die Reaktionskurve des Endproduktes $\mathrm{Fe}^{5}$ setzt verzögert ein, man erhält einen S-förmigen Kurventyp mit der Anfangssteigung 0 und einem Wendepunkt. Während bei einem Nettobedarf von einem Quant mit Einschalten der Lichtquelle die Bildung des Endprodukts sofort einsetzt; man erhält eine einfache Exponentialkurve ohne Wendepunkt mit einer von 0 verschiedenen Anfangssteigung (Abb. 1).

Eine Abschätzung für die Lage des Wendepunkts in Abb. 1, Kurve $n^{*}=2$, können wir - wenn wir von Rückreaktionen absehen - aus der Treffertheorie entnehmen:

Ist $m$ das Verhältnis der absorbierten Lichtquanten zu den vorhandenen FeCO-Gruppen, oder, anders ausgedrückt, die mittlere Belegung der FeCO-Gruppen mit Lichtquanten, dann ist die Wahrscheinlichkeit für den Anteil von FeCO-Gruppen, der durch kein Lichtquant getroffen wurde

$$
W_{0}=\frac{m^{0}}{0 !} e^{-m},
$$

durch ein Lichtquant getroffen wurde

$$
W_{1}=\frac{m^{1}}{1 !} e^{-m}
$$

und durch zwei oder mehr Lichtquanten getroffen wurde

$$
W=1-W_{0}-W_{1}=1-(1+m) e^{-m} .
$$

Hieraus erhalten wir den Wendepunkt, indem wir zweimal nach $m$ differenzieren und die 2. Ableitung gleich null setzen:

$$
W^{\prime \prime}=(1-m) e^{-m}=0 \text {; gibt } m=1 .
$$

Der Wendepunkt liegt demnach an der Stelle $m=1$, d. h. wenn die Zahl der absorbierten Lichtquanten gerade gleich der Zahl der FeCO-Gruppen ist.

\section{Versuchsanordnung}

Auf eine Meßküvette (Abb. 2), die mit der zu untersuchenden Lösung gefüllt ist, fällt das die photochemische Reaktion bewirkende Licht $\mathrm{L}_{1}$ als paralleler, monochromatischer Lichtstrahl. Als Beleuchtungsquelle dient die Quecksilberlampe HBO 200 der Firma Osram, als Filter, Farbgläser der Fa. Schott u. Gen. Der Lichtstrahl $\mathrm{L}_{1}$ kann durch Betätigen eines Kamera-Zentralverschlus-

$5 \mathrm{Fe}$ bedeute hier und im Folgenden stets das Eisen eines Häms. 
ses eingeschaltet und seine Intensität über ein Vakuumthermoelement an einem Galvanometer mit kleinem Innenwiderstand, $G_{1}$, abgelesen werden. Die Eichung des Thermoelementen-Kreises erfolgte mit einem von der Physikalisch-Technischen Bundesanstalt, Braunschweig, in bezug auf seine Gesamtstrahlung geeichten Lichtnormals Wi E 6 der Fa. Osram; die PTB verbürgt eine Genauigkeit ihrer Eichung auf $\pm 3 \%$.

Für alle Versuche wurde ein Gasgemisch von $10 \% \mathrm{CO}$ und $90 \% \quad \mathrm{O}_{2}$ in einem Vorratsbehälter aufbewahrt. Das CO-Gas wurde durch Zutropfen von Ameisensäure in warme, konzentrierte Schwefelsäure gewonnen und in Kalilauge gewaschen, das $\mathrm{O}_{2}$-Gas einer $\mathrm{O}_{2}$-Flasche entnommen. Bei den einzelnen Versuchen wurde die gewünschte Pufferlösung mit der Gasmischung vor der Hämoglobin-Zugabe gesättigt, da beim Schütteln der Hämoglobin-Lösung das Hämoglobin durch Schaumdenaturierung zerstört wird. Die Hämoglobinkonzentration in der Meßküvette betrug meistens $2-3 \cdot 10^{-8} \mathrm{Mol} \mathrm{Fe} / \mathrm{ccm}$. Durch Anwendung dieses Gasgemisches wurde - wie bei B ü c h e r ${ }^{1}$ - anstatt der Reaktion $\mathrm{FeCO}=\mathrm{Fe}+\mathrm{CO}$

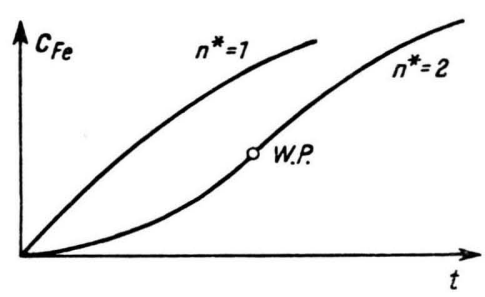

Abb. 1. Reaktionsbeginn der FeCO-Spaltung durch Licht bei einem Netto-Quantenbedarf $n^{*}=1$ und $n^{*}=2$. Zur Zeit $t=0$ wird die Lichtquelle eingeschaltet. W.P. = Wendepunkt.

die Reaktion $\mathrm{FeCO}+\mathrm{O}_{2}=\mathrm{FeO}_{2}+\mathrm{CO}$ verfolgt. Das Endprodukt $\mathrm{Fe}$ durch das Endprodukt $\mathrm{FeO}_{2}$ zu ersetzen, kann unbedenklich geschehen, da die Geschwindigkeit, mit der die Reaktion $\mathrm{Fe}+\mathrm{O}_{2} \rightarrow \mathrm{FeO}_{2}$ verläuft, bei dem angewandten $\mathrm{O}_{2}$-Druck die anderen Geschwindigkeiten der FeCO-Hell- und Dunkelreaktionen um das 100-fache und mehr übersteigt. In experimenteller Hinsicht gewinnt man damit den großen Vorteil, nicht unter vollständigem $\mathrm{O}_{2}$-Ausschluß arbeiten zu müssen.

Um die Umwandlung von CO-Hämoglobin in $\mathrm{O}_{2}-$ Hämoglobin spektrophotometrisch verfolgen zu können, tritt senkrecht zu dem die photochemische Spaltung bewirkenden Lichtstrahl $L_{1}$ ein im Verhältnis zu diesem ganz schwacher Meßlichtstrahl $\mathrm{L}_{2}$ durch die Meßküvette hindurch. Als Lichtquelle für diesen dient eine Quecksilberlampe, die durch eine Eisenwasserstofflampe stromstabilisiert wird. Für das Meßlicht wird die Wellenlänge $\lambda=366 \mathrm{~m} \mu$ benützt, da die als Empfänger vorgesehene Photozelle mit Sekundärelektronen-Vervielfacher (SEV, Fa. Maurer, Neuffen) für diese Wellenlänge besonders empfindlich ist. Der SEV wird von einem glimmlampenstabilisierten Hochspannungsgerät gespeist. Der durch den SEV verstärkte Photostrom wird von dem Galvanometer $\mathrm{G}_{2}$ angezeigt; gleichzeitig ist die Anode des SEV mit einer Platte des Y-Plattenpaares eines Kathodenstrahl-
Oszillographen verbunden. Das Schirmbild des Oszillographen kann mit einer Kamera aufgenommen werden. Der Steigerung der Empfindlichkeit des Oszillographen dient ein Oszillographenrohr mit Nachbeschleunigung, das dazu noch mit Unterspannung betrieben wird. Die dabei auftretenden, unvermeidlichen Verzerrungen stören bei Auswerten der Aufnahmen für die Bestimmung des Brutto-Quantenbedarfs nicht, weil vor jeder photographischen Aufnahme der Reaktionskurve zwei Voraufnahmen zur Abbildung eines Koordinatennetzes gemacht werden (vgl. Abb. 3): Eine Potentialdifferenz-Einstellung an den Y-Platten des Oszillographen ergibt zusammen mit der Dunkelgleichgewichts-Waagrechten der Hauptaufnahme zwei Höhenmarken, die einer bestimmten Konzentrationsverschiebung $\Delta c$ von $\mathrm{FeCO}$ zu $\mathrm{FeO}_{2}$ entsprechen. Zur Zeitmessung dient ein Zeitmarkenraster, das durch eine Röhrenkippschaltung (Multivibratorschaltung)

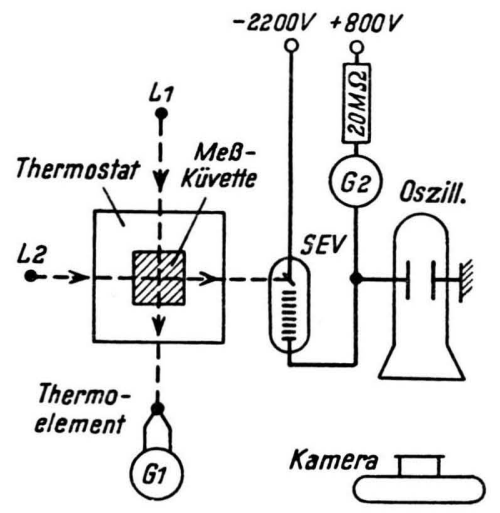

Abb. 2. Versuchsanordnung, Erklärungen s. Text.

erzeugt wird. Im hier gezeigten Beispiel einer Aufnahme (Abb. 3) entspricht dem Abstand der Höhenmarken eine Konzentrationsverschiebung $\Delta c=0,014 c$ (wo $c=$ Konzentration des gesamten Hämoglobineisens bedeutet) und dem Zeitmarkenabstand eine Zeit von 0,755 Sekunden.

\section{Darstellung des Hämoglobins und Eisenbestimmung}

Frisch entnommenes, defibriniertes Blut wurde 3-mal in 0,9-proz. NaCl-Lösung durch Zentrifugieren gewaschen. Nach der 3. Zentrifugation wurde mit 0,01-m. Boratpuffer $\left(p_{\mathrm{H}}=9\right)$ auf das 2,5-fache des ursprünglichen Blutvolumens aufgefüllt. Die Erythrocyten lysierten sofort. Das Stroma ist zum Teil so fein verteilt, daß es erst durch sehr hochtouriges Zentrifugieren abgetrennt werden kann ${ }^{6}$. Hammelhämoglobin wurde deshalb 20 Min., Pferdehämoglobin $40 \mathrm{Min}$. lang bei $25000 \mathrm{~g}$ zentrifugiert. Die so gereinigte Hämoglobin-Lösung wurde im Kühlschrank aufbewahrt. Das Hämoglobin hielt sich etwa eine Woche lang frisch, d. h. zeigte keine Beeinträchtigung in seiner Reaktionsfähigkeit. Die Reaktionsfähigkeit älterer Hämoglobin-Lösungen ließ in steigendem Maße nach.

6 G. Ruhenstroth u. G. Schumacher, Z. Naturforschg. 8 b, 512 [1953]. 
Die Mengenbestimmung des Hämoglobineisens geschah durch photometrische Titration mit Kaliumeisen(II)-cyanid bei der Wellenlänge $\lambda=630 \mathrm{~m} \mu$ im Beckmann-Spektrographen. Hierbei wird das Hämoglobin zu dem braun gefärbten Methämoglobin oxydiert. Wie $\mathrm{H}$ a v e $\mathrm{m}$ a $\mathrm{n} n$ und Mitarbb. 7 und Dru ckrey und Mitarbb. ${ }^{8}$ durch Vergleich mit manometrischen Messungen feststellten, verläuft die Reaktion bei $p_{\mathrm{H}} 7$ vollständig, d. h. ein Hämoglobineisen wird durch ein $\mathrm{K}_{3} \mathrm{Fe}(\mathrm{CN})_{6}$ oxydiert. Untersuchungen, die bei Zimmertemperatur ausgeführt wurden, ergaben, daß bei höheren $p_{\mathrm{H}}$-Werten ein großer Überschuß von $\mathrm{K}_{3} \mathrm{Fe}(\mathrm{CN})_{6}$ zur Oxydation benötigt wird, während sie bei $p_{\mathrm{H}} 5$ wie im Neutralen vollständig, aber schneller verläuft und die von Druckrey besprochene Abweichung in der Umgebung des Äquivalenzpunktes (Näheres hierüber s. 1. c. ${ }^{8}$ ) kleiner ausfiel; deshalb wurde die Titration in $0,01-m$. Phosphatpuffer bei $p_{\mathrm{H}} 5$ durchgeführt.

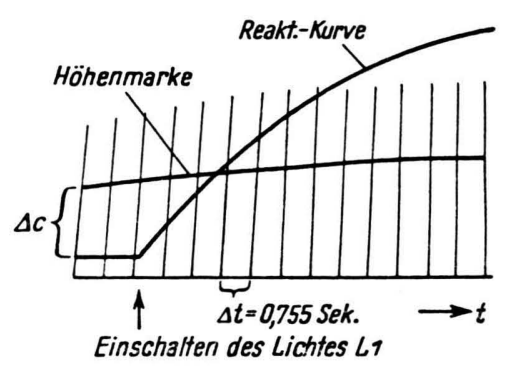

Abb. 3. Oszillographenbild einer Reaktionskurve mit Zeitmarkenraster und Höhenmarke, die - vom Dunkelgleichgewicht aus gerechnet - einer Konzentrations-Verschiebung $\Delta c=0,014 c$ entspricht. Nach photographischer Aufnahme gezeichnet.

\section{Bestimmung des Netto-Quantenbedarfs $n^{*}$}

Um eine möglichst vergrößerte Aufnahme des Reaktionsbeginns zu erhalten, wurde für eine Reihe von Aufnahmen der SEV mit einer Nachverstärkung versehen und eine schnellere Zeitablenkung am Oszillographen eingestellt. Die Wiedergabe einer solchen Aufnahme zeigt Abb. 4. Um den Öffnungsvorgang des Verschlusses darstellen zu können, wurde das Streulicht des Lichts $\mathrm{L}_{1}$ nicht wie bei den anderen Aufnahmen durch ein entsprechendes Filter vom SEV ferngehalten. Kurve a in Abb. 4 zeigt den Öffnungsvorgang allein, die Küvette ist mit $\mathrm{O}_{2}$-Hämoglobin gefüllt, bei dem keine Lichtreaktion stattfindet. Kurve b zeigt Öffnungsvorgang und Reaktionskurve, die Küvette ist mit $\mathrm{CO}-\mathrm{O}_{2}-\mathrm{Hämoglobingemisch} \mathrm{ge-}$ füllt.

Entsprechend der Kurve a können wir die Dauer des Öffnungsvorgangs mit $0,015 \mathrm{sec}$ ansetzen. Da

7 R. Havemann, F. Jung u. B. v. Is sekutz, Biochem. Z. 301, 116 [1939]. nach dieser Zeit in der Kurve b ein Wendepunkt nicht auftritt - und, wie ein Blick auf Abb. 3 lehrt, auch später als nach 0,3 sec nicht —, entnehmen wir, daß eine eventuelle Verzögerung des Reaktionsgemisches innerhalb der Öffnungszeit des Verschlusses liegen muß, also kleiner als 0,015 sec ist. Demgegenüber ist in dem Beispiel der Abb. 4 die mittlere Belegung der FeCO-Gruppen mit Lichtquanten, $m=1$, erst nach 3,4 sec erreicht, die Zeit, bei der entsprechend Abb. 1 bei einem Netto-Quantenbedarf $n^{*}=2$ der Wendepunkt in der Reaktionskurve zu erwarten wäre. Also ist der Netto-Quantenbedarf bei der FeCO-Spaltung $n^{*}=1$.

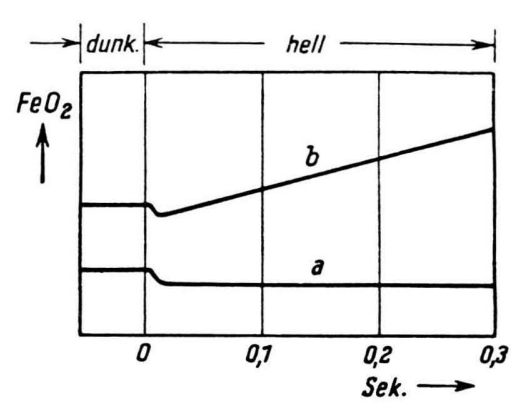

Abb. 4. Kurve a: Zeitlicher Verlauf des Öffnens des Verschlusses. Kurve b: Zeitlicher Verlauf des Beginns der FeCO-Spaltung durch Licht. Nach photographischer Aufnahme gezeichnet, Zeitmaßstab entzerrt.

Eine kritische Bemerkung ist noch zu machen. In Abb. 1 sind als Ordinate die Konzentrationsunterschiede zwischen Anfangs- und Endprodukt aufgetragen, in Abb. 3 und 4 dagegen die zugehörigen Extinktionsunterschiede. Die Darstellung der Kurve für $n^{*}=2$ in Abb. 1 gibt die Verhältnisse für Abb. 3 und 4 nur dann gültig wieder, wenn man annimmt, daß das Zwischenprodukt - es heiße $(\mathrm{FeCO})^{\prime}$ - die gleiche Extinktion wie das Ausgangsprodukt FeCO hat. Sollte das Zwischenprodukt (FeCO)' bei der betreffenden Wellenlänge des Meßlichts $\mathrm{L}_{2}$ zufälligerweise die gleiche Extinktion wie das Endprodukt $\mathrm{FeO}_{2}$ haben, dann ist bei der Extinktionsmessung nur der erste photochemische Schritt $\mathrm{FeCO} \rightarrow(\mathrm{FeCO})^{\prime}$ erkennbar, aber nicht der zweite photochemische Schritt $(\mathrm{FeCO})^{\prime} \rightarrow \mathrm{FeO}_{2}$, man erhält bei der Extinktionsmessung keine S-förmige Kurve mehr, sondern eine einfache Exponentialkurve; ein Netto-Quantenbedarf $n^{*}=1$, der daraus folgt, wäre also ein für die Gesamtreaktion vorgetäuschter.

Wenn man sicher gehen will, keiner derartigen Täuschung erlegen zu sein, ist die Messung bei verschiedenen Wellenlängen des Meßlichts durchzuführen. Ich habe deshalb bei den Aufnahmen als Meßlicht $\mathrm{L}_{2}$ neben der er-

8 H. Druckrey, P. D a n neberg, H. K a i s e r, I. From m e u. H. S ch ne id e r, Biochem. Z. 322, 535 [1952]. 
wähnten Hg-Linie $366 \mathrm{~m} \mu$ auch die Linie $578 \mathrm{~m} \mu$ verwandt. Beide Typen von Aufnahmen lassen keinen Wendepunkt außerhalb der Einschaltzeit erkennen.

\section{Bestimmung des Brutto-Quantenbedarfs $n$}

Nach W a rburg ${ }^{2}$ erhält man den Brutto-Quantenbedarf $n$ aus der Zeitkonstante des Reaktionsverlaufs im Hellen, verglichen mit der Zeitkonstante der Rückreaktion im Dunkeln. Dabei sind die Bedingungen einzuhalten, daß die Lösung Hämoglobin nur in so geringen Mengen enthält, daß die Lichtintensität beim Durchgang durch die Lösung nur wenig durch Absorption geschwächt wird, und daß die CO-Konzentration so sehr die Hämoglobin-Konzentration übersteigt, daß sie während des ganzen Versuchs bei dem ja CO frei wird - trotzdem als konstant betrachtet werden kann.

Der Brutto-Quantenbedarf $n$ kann aber auch aus der Anfangssteigung der Reaktionskurve durch eine photographische Aufnahme der Art von Abb. 3 abgelesen werden. Nach diesem Verfahren sind die in Tab. 2 und 3 aufgeführten Meßergebnisse gewonnen worden. Meßtechnisch vorteilhaft hierbei ist, daß die bei $\mathrm{W}$ arburg notwendige Bedingung der geringen Hämoglobin-Konzentration und der großen CO-Konzentration nicht erfüllt sein muß. Dies erlaubt gegenüber Warburg die Benutzung einer kleinen Meßküvette mit konzentrierter HämoglobinLösung und eine entsprechend starke Bündelung des Lichts $\mathrm{L}_{1}$. Demgegenüber hat $\mathrm{W}$ a r b u r g s Methode den Vorzug, einen Überblick über den Gesamtablauf der Reaktion zu geben.

Die Formel für $n$ werde zunächst für den Fall einer so schmalen Küvette hergeleitet, daß der Weg des Lichts durch die Küvette so klein ist, daß die Intensität $J$ des einfallenden Lichts beim Durchgang durch die Küvette kaum geschwächt wird, und sie für alle Stellen der Küvette als konstant angesehen werden kann.

Ist $c_{1}$ und $c_{2}=$ Konz. des FeCO bzw. des $\mathrm{FeO}_{2}$, insbesondere

$$
\begin{aligned}
c_{10} \text { und } c_{20}= & \begin{array}{l}
\text { Konz. des } \mathrm{FeCO} \text { bzw. des } \mathrm{FeO}_{2} \text { des } \\
\text { Dunkelgleichgewichts, }
\end{array} \\
\beta_{1} \text { und } \beta_{2}= & \underset{\text { Absorptionskoeffizient des } \mathrm{FeCO} \text { bzw. }}{ } \mathrm{FeO}_{2},
\end{aligned}
$$

dann ist beim Einschalten des Lichts die FeCO-Konzentration $c_{10}$ und die je Zeiteinheit vom FeCO absorbierte Lichtmenge $\beta_{1} J c_{10}$. Hiervon ist der Bruchteil $1 / n$ photochemisch wirksam, also haben wir im Anfang nach dem Einschalten des Lichts einen photochemischen Umsatz je Zeiteinheit

$$
\left(\frac{\mathrm{d} c}{\mathrm{~d} t}\right)_{0}=\frac{\beta_{1} J c_{10}}{n} \text { oder es ist } n=\frac{\beta_{1} J c_{10}}{(\mathrm{~d} c / \mathrm{d} t)_{0}}
$$

Für den Übergang auf beliebig breite Küvetten sei: $J_{0}=$ Intensität des Lichts an der Lichteintrittsstelle,

$J_{1}=$ Intensität des Lichts an der Lichtaustrittsstelle,

$x=$ beliebige Stelle des Lichtweges in der Küvette und

$l=$ gesamte Weglänge des Lichts in der Küvette.

Dann ist die Lichtintensität an der Stelle $x$ zur Zeit $t=0$ (Einschalten des Lichts) gegeben durch:

$$
J=J_{0} e^{-\left(\beta_{1} c_{10}+\beta_{2} c_{20}\right) x} .
$$

Dies in die Formel für $(\mathrm{d} c / \mathrm{d} t)_{0}$ eingesetzt, gibt:

$$
\left(\frac{\mathrm{d} c}{\mathrm{~d} t}\right)_{0}=\frac{\beta_{1} c_{10}}{n} \cdot J_{0} e^{-\left(\beta_{1} c_{10}+\beta_{2} c_{20}\right) x} .
$$

Hiervon der Mittelwert gebildet, gibt:

$$
\begin{gathered}
\overline{\left(\frac{\mathrm{d} c}{\mathrm{~d} t}\right)_{0}}=\frac{\beta_{1} c_{10}}{n l} \cdot \int_{0}^{l} J \mathrm{~d} x=\frac{\beta_{1} c_{10}\left(J_{0}-J_{1}\right)}{n l\left(\beta_{1} c_{10}+\beta_{2} c_{20}\right)}= \\
=\frac{J_{0}-J_{1}}{n l\left(1+\beta_{2} c_{20} / \beta_{1} c_{10}\right)} .
\end{gathered}
$$

Daraus erhält man die Endformel:

$$
n=\frac{J_{0}-J_{1}}{\overline{(\mathrm{d} c / \mathrm{d} t)_{0}}\left(1+\beta_{2} c_{20} / \beta_{1} c_{10}\right) l} .
$$

Auf einen Umstand soll noch aufmerksam gemacht werden: $c$ wird spektrophotometrisch bestimmt, d. h. durch Absorptionsmessung des Lichts im Lichtweg 2. Bei konzentrierten Hämoglobin-Lösungen nimmt die Lichtintensität beim Durchgang durch die Küvette so sehr ab, daß in der Nähe der Lichteintrittsstelle - wegen der größeren Lichtintensität - im allgemeinen mehr FeCO gespalten sein wird als in der Nähe der Lichtaustrittsstelle, so daß die Lösung nicht mehr als homogen angesehen werden kann. Bei Lösungen mit ungleicher KonzentrationsSchichtung und einem Verlauf des Meßlichts längs der Schichtflächen - wie dies hier der Fall ist - ist das arithmetische Mittel über die Konzentrationen nur dann proportional dem arithmetischen Mittel über die hindurch gelassenen Lichtintensitäten, wenn die Konzentrationsunterschiede sehr gering sind. Gerade dies trifft aber bei Beginn der Lichtreaktion noch zu, weil der Reaktionsablauf mit der überall gleich großen Dunkelgleichgewichts-Konzentration beginnt. Damit ist die Anfangssteigung der Reaktionskurve, wie sie auf dem Oszillographen-Schirmbild erscheint, der mittleren Konzentrationsänderung $(\mathrm{d} c / \mathrm{d} t)_{0}$ proportional, und die Auswertung der Aufnahmen ist ebenso einfach wie bei homogenen Lösungen.

In Tab. 2 und 3 sind die Meßergebnisse des BruttoQuantenbedarfs als Durchschnittswerte der jeweiligen Aufnahmereihen wiedergegeben. Die Ungenauigkeit der Werte kann bis etwa $\pm 10 \%$ betragen. Ein Vergleich der Tab. 2 mit den in Tab. 1 zusammengefaßten Werten von B ü cher zeigt eine der Meßgenauigkeit entsprechende Übereinstimmung. 


\begin{tabular}{|c|c|c|c|}
\hline$p_{\mathrm{H}}$ & Puffer & $\begin{array}{c}\text { Brutto-Quante } \\
\text { Hammel- } \\
\text { CO-Häm }\end{array}$ & $\begin{array}{l}\text { bedarf } n \text { bei } \\
\text { Pferde- } \\
\text { lobin }\end{array}$ \\
\hline 9 & 0,01-m. Borat & 4,3 & 4,8 \\
\hline 7 & 6-m. Harnstoff & 3,4 (undiss.) & 2,2 (diss.) \\
\hline 5 & 0,01-m. Phosphat & 3,9 & 5,0 \\
\hline
\end{tabular}

Tab. 2. Brutto-Quantenbedarf $n$ bei verschiedenen Wasserstoffionen- und Salzkonzentrationen. CO-Hammel- und Pferde-Hämoglobin. Wellenlänge des spaltenden Lichts $\lambda=546 \mathrm{~m} \mu$. Temperatur $4^{\circ} \mathrm{C}, c_{10} / c=83$ Prozent.

\begin{tabular}{|c|c|c|}
\hline Temperatur & $\mathrm{c}_{10} / \mathrm{c}[\%]$ & Bruttoquantenbedarf $n$ \\
\hline $4^{\circ} \mathrm{C}$ & 83 & 4,3 \\
$20^{\circ} \mathrm{C}$ & 83 & 3,7 \\
$4^{\circ} \mathrm{C}$ & 10 & 3,7 \\
\hline
\end{tabular}

Tab. 3. Brutto-Quantenbedarf $n$ bei verschiedenen Temperaturen und Ausgangskonzentrationen des FeCO. COHammelhämoglobin. 0,01-m. Boratpuffer $p_{\mathrm{H}}$ 9. Wellenlänge des spaltenden Lichts $\lambda=546 \mathrm{~m} \mu$.

$\mathrm{Zu}$ den Werten von Tab. 2 sei noch Folgendes bebemerkt: Allgemein wird angenommen (s. z. B. 9), daß Pferdehämoglobin in verdünnten Lösungen (unterhalb $1 \%$ ) in Halbhämoglobin zerfällt, wie dies in den stark salzhaltigen Lösungen der Fall ist. Dieser Annahme liegen aber nur Messungen älteren Datums zugrunde, die bisher nicht überprüft worden sind: nach osmotischen Messungen bestimmte $\mathrm{R} \mathrm{oche}$ und $\mathrm{Ada}$ ir ${ }^{9}$ ein Mol.Gew. von 33400 , Diffusionsmessungen von $\mathrm{T}$ is e li us und G r o s s 10 ergaben eine mit fortschreitender Verdünnung bis auf das 4-fach ansteigende Diffusionskonstante, während $\mathrm{P}$ e d e r s e $\mathrm{n}$ ein Absinken der Sedimentationskonstante beobachtete, unveröffentlicht, s. l. c. 11).

Unsere, noch mehr B ü c her s Messungen wurden bei Konzentrationen weit unterhalb 1\% gemacht. Die Messungen des Brutto-Quantenbedarfs bei Hammelhämoglobin, das nicht dissoziiert, ergeben beim Übergang von 0,01-m. Pufferlösung zu starker Salzkonzentration nur einen geringen Salzeffekt. Man müßte deshalb beim Pferdehämoglobin, wenn es bei starker und bei schwacher Salzkonzentration dissoziiert wäre, erwarten, daß es außer einem kleinen Salzeffekt keinen Unterschied im Quantenbedarf zeigt. Das trifft nicht zu. Der Quantenbedarf des Pferdehämoglobins ist sogar in 0,01- $m$. Pufferlösung um ein Geringes größer als beim Hammelhämoglobin; allein das Pferdehämoglobin in starker Salzlösung, für das die Dissoziation in Halbhämoglobin sichergestellt ist ${ }^{4}$, zeigt den auffallend niederen Quantenbedarf. Ich

$9 \mathrm{~J}$. W y m a n, Advances in Protein Chemistry IV, 407 [1948].

10 A. Tis e lius u. D. Gross, Kolloid-Z. 66, 11 [1934].

\begin{tabular}{|c|c|c|c|c|}
\hline \multicolumn{2}{|c|}{$p_{\mathrm{H}}=7$} & \multirow{2}{*}{$\begin{array}{c}\omega \\
\left(5^{\circ} \mathrm{C}\right) \\
\sec ^{-1}\end{array}$} & \multirow{2}{*}{$\left(\begin{array}{c}\omega \\
\left(20^{\circ} \mathrm{C}\right) \\
\sec ^{-1}\end{array}\right)$} & \multirow{2}{*}{$\begin{array}{c}\mathrm{W}^{*} \\
\mathrm{cal} / \\
\mathrm{Mol}\end{array}$} \\
\hline Puffer & Hämoglobin von & & & \\
\hline 0,01-m.Phosphat & Hammel(undiss.) & 0,0073 & 0,039 & 19500 \\
\hline $0,01-m$. Phosphat & Pferd (undiss.) & 0,0047 & 0,029 & 21500 \\
\hline 6-m. Harnstoff & Hammel (undiss.) & 0,0116 & 0,060 & 17300 \\
\hline 6-m. Harnstoff & Pferd (diss.) & 0,0040 & 0,039 & 24500 \\
\hline
\end{tabular}

Tab. 4. Reaktionsgeschwindigkeit $\gamma=\omega$ und Aktivierungsenergie $W^{*}$ des FeCO-Zerfalls im Dunkeln.

vermute deshalb, daß Pferdehämoglobin in stark verdünnten, aber salzarmen Lösungen nicht in Halbhämoglobin dissoziiert.

Tab. 3 zeigt, daß ein Gang mit der Temperatur oder mit der Ausgangskonzentration nicht besteht, oder so klein ist, daß er noch innerhalb der Fehlergrenzen der Messungen liegt.

Der Variation des Verhältnisses $c_{10} / c$ liegt folgende Bedeutung zugrunde: Bei $c_{10} / \mathrm{c}=83 \%$ handelt es sich vorwiegend um mit CO-abgesättigtes Hämoglobin, d. h. um Hämoglobin, dessen $4 \mathrm{Fe}$ alle mit $\mathrm{CO}$ besetzt sind; während beim Verhältnis $c_{10} / c=10 \%$ bei der überwiegenden Mehrzahl von HämoglobinMolekülen nur $1 \mathrm{Fe}$ mit $\mathrm{CO}$ besetzt ist. Tab. 3 zeigt also, daß die Besetzungszahl der Hämoglobin-Moleküle mit CO für den Brutto-Quantenbedarf keine Rolle spielt.

\section{Die Aktivierungsenergie der FeCO-Spaltung}

Die Aktivierungsenergie der FeCO-Spaltung ist beim CO-Hämoglobin von $\mathrm{H}$ artridge und Roughton ${ }^{12}$ zu etwa $21000 \mathrm{cal} / \mathrm{Mol}$ bestimmt worden.

Bei Zugrundelegung eines Nettobedarfs von einem Quant je Reaktion liefert 1 Mol Lichtquanten des spaltenden Lichts $(\lambda=546 \mathrm{~m} \mu) 52100$ cal, also eine für den Spaltungsprozeß ausreichende Energiemenge.

Der Bestimmung der Aktivierungsenergie für Halbhämoglobin erfolgte nach eigenen Messungen (siehe Tab. 4), zur Kontrolle sind die Messungen auch auf Hämoglobin ausgedehnt worden. Die Werte für gewöhnliches Hämoglobin stimmen mit den von $\mathrm{Hartridge}$ und Roughton gemessenen gut überein. Eine energetische Besonderheit für Halbhämoglobin liegt nach Tab. 4 nicht vor.

11 T. S v e d b e r g, Die Ultrazentrifuge, Leipzig 1940.

$12 \mathrm{H}$. Hartridge u. J. W. Roughton, Proc. Roy. Soc. [London] Ser. A 104, 395 [1923]. 
Die Aktivierungsenergie der FeCO-Spaltung kann man wie folgt erhalten: Die Aktivierungsenergie ist:

$$
W^{*}=R \frac{\ln \gamma_{1}-\ln \gamma_{2}}{1 / T_{1}-1 / T_{2}},
$$

wo $T_{1}$ und $T_{2}$ zwei absolute Temperaturen bedeuten und $\gamma_{1}$ und $\gamma_{2}$ die bei diesen Temperaturen gemessenen Geschwindigkeiten der Reaktion $\mathrm{FeCO} \underset{\rightarrow}{\longrightarrow} \mathrm{Fe}+\mathrm{CO}$ im Dunkeln. Da die Geschwindigkeit $\gamma$ im FeCO- Fe- $\mathrm{FeO}_{2}-$ System die weitaus kleinste ist, ist sie für den Verlauf der Bruttoreaktion $\mathrm{FeCO} \stackrel{\omega}{\longrightarrow} \mathrm{FeO}_{2}$ geschwindigkeits-bestimmend, das heißt, es ist $\omega=\gamma . \omega$ kann man aus dem Gleichgewichtswert der FeCO-Konzentration $c_{10}$ und der Zeitkonstante $\alpha$, mit der ein nicht im Gleichgewicht befindliches $\mathrm{FeCO}-\mathrm{FeO}_{2}$-System in die Gleichgewichtslage übergeht, durch $\omega=\alpha\left(1-c_{10} / c\right)$ bestimmen. Eine Gleichgewichtsstörung wird am einfachsten durch Belichten hergestellt.

\section{Schlußbetrachtung}

Wenn auch über die Ursache, warum das Hämoglobin und Halbhämoglobin einen höheren BruttoQuantenbedarf beim Spaltungsprozeß haben als andere Häm-Verbindungen, nichts gesagt werden kann, so läßt sich doch noch ein Hinweis über die Gleichartigkeit der Beteiligung der verschiedenen Häme des Hämoglobin-Moleküls an der Spaltungsreaktion geben.

Aus der Tatsache, daß sich die Reaktionskurve während der Belichtung als einfache Exponentialkurve darstellen läßt ${ }^{1}$, geht hervor, daß sich alle photochemisch empfindlichen FeCO-Stellen in bezug auf die Lichtabspaltung des $\mathrm{CO}$ reaktionskinetisch gleich verhalten.
Damit ist noch offen gelassen, ob es vielleicht beim Hämoglobin und Halbhämoglobin neben photochemisch wirksamen FeCO-Stellen auch photochemisch unwirksame FeCO-Stellen gibt. Mit dieser Annahme wäre die auffallende Größe des Brutto-Quantenbedarfs $n$ erklärbar, da er sich ja auf die Lichtabsorption an allen FeCO-Stellen bezieht. Trotzdem möchte ich diesen Fall ausschließen. Eine Blockierung der photochemischen Wirksamkeit setzt an den betreffenden Stellen einen tiefer greifenden Strukturwandel der Umgebung voraus, so daß sich die photochemisch wirksamen von den blockierten FeCO-Stellen auch im sonstigen chemischen Verhalten reaktionskinetisch unterscheiden müßten. Untersuchungen am Hämoglobin ergeben aber, daß alle vier Fe-Stellen ein chemisch gleiches Verhalten in bezug auf die Abhängigkeit vom $p_{\mathrm{H}}$-Wert als auch von der Temperatur zeigen (s. 1. c. ${ }^{9}$ S. 445), d. h. daß sowohl die für die $\mathrm{O}_{2}$ - und CO-Reaktionen maßgebliche Verteilung der Ladungen sowie der Energiepotentiale in der Umgebung aller vier Hämorte am Hämoglobinmolekül gleich sind. Ich möchte deshalb auch für den photochemischen Spaltungsprozeß ein gleichartiges Verhalten an allen FeCO-Stellen annehmen.

Danach ergibt sich das Bild, daß an allen FeCOStellen der Netto-Quantenbedarf $n^{*}=1$ ist, der Bruttoquantenbedarf $n$ zwar größer als 1 , aber für alle FeCO-Stellen gleich ist.

Herrn Prof. Dr. H. Friedrich-Freks a sei auch an dieser Stelle für Anregungen und sein stets förderndes Interesse an der Arbeit gedankt. 